\title{
SENSIBILITÉ À LA DOULEUR ET COMPASSION CHEZ ROBERT CHALLE : DE L'EXPÉRIENCE DE L'ATTENDRISSEMENT À UNE RÉFLEXION SUR LA NATURE HUMAINE
}

\author{
CAMILLE GUYON-LECOQ \\ Université de Picardie Jules Verne
}

\section{SENSITIVITY TO PAIN AND COMPASSION IN ROBERT CHALLE'S WRITINGS: FROM THE EXPERIENCE OF "SOFTENING OF THE HEART" TO A REFLECTION ON HUMAN NATURE}

\begin{abstract}
This paper assumes that the most vivid existential experience that Challe had during the voyage he reported in the Journal d'un voyage fait aux Indes orientales (The Diary of a Voyage to the East Indies) was the experience of pain and the spectacle of pain. It shows that the lyrical idea of "softening of the heart" endowing sensitivity with the statute of human disposition for virtue par excellence inspires and feeds the writing of the voyage. By a reflection on the various figures of "softening of the heart" described and staged in the Diary it intends to shed some light not only on the anthropological but also on the metaphysical discoveries of a traveller nurtured by operas such as Challe. He can be seen, day after day, outlining a new conception of the idea of nature thanks to accidental experiences always confronted with the learning from books in which his sensitivity to the lyrical art may take a part.
\end{abstract}

Keywords: Robert Challe, pain, compassion, sensitivity, human nature, "softening of the heart", lyrical art

Mots clés : Robert Challe, douleur, compassion, sensibilité, nature humaine, attendrissement, art lyrique

S'il est des hommes qui embarquent pour apprendre à connaître le monde, s'il en est d'autres qui embarquent pour apprendre à se connaître eux-mêmes, Robert Challe, pour avoir embarqué, n'apprit pas seulement à mieux connaître un monde, tant naturel qu'humain comme élargi aux frontières de l'aventure, ni à mieux se connaître lui-même. Sa singularité fut surtout d'avoir appris à connaitre les autres hommes de sa sorte, embarqués eux aussi, avec lui ou sur d'autres bateaux, tant amis qu'ennemis, et à approcher par là ce qui fait l'homme, objet, tant dans Les Illustres Françaises ${ }^{1}$ et dans les Difficultés sur

1 Challe, R. (1959) : Les Illustres Françaises. Édition établie par F. Deloffre. Paris : Les Belles Lettres, 2 vol., $3^{\mathrm{e}}$ tirage, 1973. 
la religion proposées au Père Malebranche ${ }^{2}$ que dans le Journal d'un Voyage fait aux Indes Orientales $^{3}$, de la curiosité inlassable de cette personnalité en qui ne sont jamais disjoints (et volontairement) l'homme, l'écrivain et le philosophe, dans quelque genre qu'il écrive.

À dire vrai, il y a dans l'œuvre de Robert Challe, un lien intrinsèque entre apprendre à se connaître soi-même d'une part et, de l'autre, apprendre à connaître les autres hommes proches de soi et, à cette occasion, apprendre ce qu'est l'homme. En effet, l'expérience existentielle sans doute la plus forte qu'ait faite Challe en voyage, c'est celle de la douleur : sa douleur propre, mais aussi la douleur des autres, et plus encore sa douleur de voir les autres dans la douleur. Cette sensibilité à la douleur, il la découvrit conjointement de manière indissolublement personnelle (et même égoïste) et altruiste, et ceci sous deux espèces. En premier lieu, celle de la terreur de la douleur qu'il pourrait être exposé à ressentir, au point qu'il lui arriva de tenir ses pistolets chargés dans la crainte du naufrage : plutôt le suicide que la noyade. Cette terreur de la douleur, si elle est terreur raisonnable, est aussi terreur d'imagination. En second lieu, celle d'une compassion vraie, profonde, sincère, ou plutôt celle d'un " attendrissement ", pour employer le vocabulaire précis de Challe, issu du registre compassionnel de l'opéra, modèle littéraire qui informe, sur ce point important dans l'ordre de l'histoire des idées, la réécriture du voyage, entre l'expérience existentielle princeps et sa publication comme œuvre. Attendrissement pour autrui qui est en soi, pour Challe, émotion aussi, et même douleur, pour celui qui compatit, rendu plus vulnérable - corps et cœur tout ensemble - du fait même qu'il s'attendrit. On pourrait dire, en termes simples, que l'attendrissement pour autrui nourrit, chez Challe, un attendrissement sur soi qui n'est pas une passion, mais une vertu et même un devoir.

Banalité, sans doute, pour les modernes que nous sommes, rompus à manier les systèmes de la sympathie, les mécanismes psycho-physiologiques de l'empathie et plus récemment les théories du care, mais découverte progressive - émouvante, et même faite pour émouvoir - sous la plume d'un Challe qui, à l'extrême fin du XVII e siècle, trouve dans les périls et les expériences du voyage, le moyen de contester, en Moderne, tout ensemble, d'une part une conception qu'on pourrait dire " catholique orthodoxe » de la compassion, d'autre part une conception rationaliste, qu'elle soit ou non strictement chrétienne, de la nature humaine.

Challe conteste ainsi, premièrement, une conception dans laquelle pour être vertu et non seulement passion, la compassion doit nécessairement s'accompagner des œuvres de charité qui empêchent le bon chrétien de céder aux charmes vénéneux de l'attendrissement sur soi, porte ouverte à la « délectation morose » qui peut nous exposer à ce péché aussi délicieux que trompeur qui serait de prendre l'abandon à la passion pour l'exercice de la vertu. Deuxièmement, en bon déiste qu'il est et instruit par une authentique réflexion sur l'idée de compassion - réflexion sur l'expérience aussi bien que réflexion religieuse, voire théologique -, et aussi par la rencontre fortuite avec certains animaux nouveaux, Challe conteste une conception de la nature qui fait à l'homme une place par-

2 Challe, R. (2000) : Difficultés sur la religion proposées au Père Malebranche. Édition nouvelle d’après le manuscrit complet et fidèle de la Staatsbibliothek de Munich par F. Deloffre et F. Moureau. Genève : Droz.

3 Challe, R. (1983) : Journal d'un Voyage fait aux Indes Orientales. Édition établie par F. Deloffre et M. Menemencioglu. Paris : Mercure de France, 2 vol. 
ticulière dans la Création et lui accorde, à lui seul, ce qu'on pourrait appeler - quand il ne le fait guère lui-même - une " âme ».

L'expérience de la douleur et du spectacle de la douleur faite au cours du voyage et par le voyage en tant qu'il propose des expériences inédites, ouvre ainsi sur des découvertes non seulement anthropologiques, mais encore métaphysiques.

L'hypothèse que nous faisons consiste à tenir cette « sensibilité à la douleur » (souvent remarquée par les critiques depuis la redécouverte de Challe par Jean Mesnard, Frédéric Deloffre et François Moureau) non pas pour une découverte anecdotique que Challe ferait, dans le voyage, au sujet de son propre "tempérament », pour prendre un terme qui appartient encore, à cette date, à la médecine des humeurs, mais, plus profondément, pour l'occasion de la découverte d'une idée qui sera centrale non seulement dans les Illustres Françaises mais dans la littérature comme dans la pensée du tournant du siècle : c'est la capacité à s'attendrir qui fait l'homme. La disposition à l'attendrissement comme la capacité à s'attendrir deviennent, dans une morale conçue comme naturelle, une valeur, et la sensibilité se trouve, de ce fait, érigée au statut de disposition par excellence de l'humanité à la vertu.

Mais le voyage ouvre aussi sur une découverte plus troublante que les Difficultés sur la Religion proposées au Père Malebranche ne faisaient que pressentir : la capacité d'attendrir et de s'attendrir n'est pas réservée à l'homme. Cette expérience et la réflexion qu'elle entraîne et à laquelle elle engage fermement les lecteurs ouvrent sur une dissolution de l'idée même d'une spécificité de la nature humaine, à l'occasion et au profit d'une nouvelle conception de l'échelle des êtres dans la nature. En somme, l'ébauche, grâce à l'expérience de l'attendrissement à l'égard d'animaux que seul le voyage pouvait faire découvrir dans leur « état de nature », d'une conception nouvelle de l'idée de nature.

Nous proposons donc un parcours dans l'image que donne le Journal d'un Voyage de l'idée d'attendrissement dont nous avons voulu l'esprit accordé à celui de Robert Challe, cet homme assez singulier, capable de garder la fraîcheur d'esprit d'un enfant de sept ans pour approfondir des réflexions philosophiques ou authentiquement théologiques. Notre itinéraire sera donc à mi-chemin d'un livre de contes pour enfants ou encore du sommaire de l'Histoire générale des Isles... ${ }^{4}$ du Père Jean-Baptiste Du Tertre et d'un traité. Traité de morale, de philosophie ou de théologie, il n'importe : un peu tout cela ensemble, comme faisait Challe, surtout dans cette écriture libre du voyage qui rappelle celle des Difficultés ou des Mémoires ${ }^{5}$.

4 Du Tertre J.-B. (1654) : Histoire générale des Isles de Saint-Christophe, de la Guadeloupe, de la Martinique et autres dans l'Amérique. Paris : J. et E. Langlois. Les titres de chapitres sont, dans la IVe partie, autant de noms d’animaux : I Traité Des poissons, II Traité Des animaux de l'air, III Traité Des animaux de la terre, pp. 257-392.

5 Challe, R. (1996) : Mémoires. Correspondance complète. Rapports sur l'Acadie et autres pièces. Éd. par F. Deloffre avec la collaboration de J. Popin. Genève : Droz. 


\section{Les « Petits poissons volants » ou Mirabilia et polémique religieuse}

L'expérience du voyage est indissociable de l'expérience d'un réel inconnu, chez Challe, comme chez la plupart des voyageurs du temps. Mais ce qui est singulier, c'est que, chez notre aventurier, l'intérêt du naturaliste pour la découverte d'espèces nouvelles ne s'arrête pas à un discours convenu sur les mirabilia, mais ouvre directement sur un exercice de la compassion active qui suscite lui-même une réflexion religieuse et même théologique. Le meilleur exemple, dans le Journal..., est l'épisode des " petits poissons volants ", précisément parce qu'avoir de la compassion pour les poissons n'est pas chose si commune et que réfléchir sur la fragilité de l'existence humaine à partir de la compassion pour les poissons est encore plus rarement attesté.

Challe découvre en voyage une espèce de poissons dont il ne soupçonnait pas l'existence, les « petits poissons volants ». Il décrit précisément la forme, la taille, la couleur tant extérieure qu'intérieure de ce poisson, sa vitesse, son activité, son lieu de vie et compare l'inconnu au connu pour faire mieux voir ce qu'il a d'extraordinaire ; il en a visiblement examiné d'assez près les ailes (en réalité des «nageoires [...] longues et revêtues d'un cartilage fort mince $\left.^{6} »\right)$ pour préciser les modalités de son vol (le cartilage doit être humide pour " porte $[\mathrm{r}]^{7} »$ ), vol dont il a mesuré la longueur ( deux cents pas $\left.{ }^{8} »\right)$; il commente précisément, en gourmet, l'usage gastronomique qu'on peut faire de ce poisson, détaillant les sauces qui permettent de valoriser sa chair ; il s'attarde enfin un instant à préciser de quels animaux il est la proie, les « bonites » et plus particulièrement les oiseaux, dont il détaille en une vignette rapide mais très suggestive quel carnage ils font de ces " petits poissons volants ». Ce petit animal est facile à " chasser ${ }^{9}$ » : il se prend dans les voiles et tombe aux pieds des matelots comme une bénédiction du ciel et de la mer, telle la manne céleste envoyée par Dieu à ses créatures :

Nous sommes remplis de poissons volants, qui se jettent dans nos voiles. Ils tombent sur le pont en telle quantité, que l'équipage en a presque autant qu'il lui en faut pour un repas, toutes les vingt-quatre heures. [...] On ne le pêche point, il vient de lui-même se jeter dans les voiles, d'où il tombe et meurt dans le moment, comme tout autre poisson de la mer, sitôt qu'il en est dehors ${ }^{10}$.

Après deux pages de description de l'animal avec la précision du naturaliste et le souci conjoint de l'homme d'action et du gastronome, on voit, non sans surprise, le discours de Challe changer complétement de direction et quitter résolument le terrain des mirabilia pour s'orienter tout soudain des joies d'une chasse ô combien aisée à l'idée de compassion :

Cette chasse est assez divertissante, mais elle ennuie à la continue, surtout ceux qui ont une espèce de compassion des petits de tous genres, qui sont toujours la proie des plus forts ${ }^{11}$.

\footnotetext{
6 Journal..., éd. cit., I, p. 150.

7 Ibidem.

8 Ibidem.

9 Ibidem.

10 Ibidem.

11 Ibid., p. 151.
} 
Le plaisir d'une " chasse » trop simple est précisément ce qui trouble les âmes sensibles : trop facile, trop inégale en fait, elle porte à la compassion. Mieux encore, cette compassion ouvre elle-même sur un débat d'ordre religieux. Assez curieusement, comme s'il était naturel de penser à la vie des hommes en observant celle des gros et des petits poissons, Challe voit dans la voracité des bonites à l'égard des poissons volants, l'image même de la barbarie des hommes qui s'entretuent " quoiqu'ils soient de même espèce ${ }^{12}$ ". De son côté, le ministre du culte, présent à bord, propose très classiquement de lire dans la destinée de ces petits poissons qui servent de nourriture aux gros poissons nommés bonites et aux oiseaux une image de l'harmonie de la nature dont la perfection a été voulue par la Providence divine, dans laquelle se manifestent et sa puissance et sa miséricorde :

Elle donne même des sujets de méditations sur le Monde et l'Eternité. Sur le monde, par rapport à la manière dure et barbare, dont les hommes en usent entre eux, quoiqu'ils soient de même espèce. Nous avons parlé là-dessus Monsieur Guisain, l'un de nos missionnaires, et moi. Il m'a dit, qu'il regardait ce poisson, que Dieu faisait naitre pour la nourriture des autres, du même point de vue que les insectes qui naissaient dans les campagnes et les bois, pour la nourriture des oiseaux ${ }^{13}$.

Mais Challe, en bon déiste, s'il s'accorde à lire, comme pourrait le faire un bon chrétien, les signes par lesquels Dieu manifeste sa présence dans une Nature que le missionnaire conçoit comme création continuée - idée à laquelle Challe ne souscrit pas plus ici que dans sa polémique avec Malebranche sur les causes occasionnelles dans les Difficultés -, propose cependant une autre lecture que le ministre du culte de ces Mirabilia. Lecture morale, sans conteste, mais on observera surtout qu'en cette occurrence (comme dans les Difficultés proposées au Père Malebranche où il trace une image proprement humaine de la compassion, essentiellement distincte de la miséricorde divine) Challe s'attache plus précisément à une « réflexion » dans laquelle le souci de l'homme est central.

J'ai là-dessus fait une autre réflexion. Je lui ai répondu que je croyais bien plutôt que ce poisson nous présentait par son infortune une vive image de nous-mêmes, par rapport à la vie et à l'Éternité, en nous instruisant que tant que nous jouissons de la vie, nous sommes toujours en danger de la perdre. Ce qui nous est figuré par ce petit animal, qui est toujours en risque dans l'eau et dans l'air : que l'eau nous indique le monde et l'air l'Eternité, qui peut ne nous être pas plus favorable ${ }^{14}$.

En termes théologiques, Challe exprime ici in fine son scepticisme de principe à l'égard de l'apocatastase. En termes simples : nous ne pouvons jamais être certains que nous serons sauvés. On sent ici, en Challe voyageur, l'unité de l'homme d'action qui se nourrit de l'expérience quotidienne du nouveau offert par le voyage, du philosophe qui appréhende ce réel en croyant sincère qui ne se juge cependant pas contraint de penser comme le ministre du culte, enfin en littérateur en puissance capable de faire d'une observation de naturaliste en herbe une petite scène plaisante. On notera surtout que l'attention portée aux mirabilia est absolument dénuée de la moindre fascination pour un quelconque

12 Ibidem.

13 Ibidem.

14 Ibidem. 
exotisme. L'expérience de l'inconnu n'est pas seulement réduite au connu ; elle est incontinent ramenée à cette quête constante de Challe : préciser ce que c'est que l'homme. Et il n'est pas indifférent que cette réflexion émane d'un sentiment, aussi fort que surprenant, de compassion à l'égard des " petits poissons volants » où il voit, selon l'heureuse formule de F. Deloffre, " une nouvelle figure de l'infini pascalien ${ }^{15}$ » : ce qui fait l'homme, en effet, pour Challe, c'est d'abord précisément la capacité de s'attendrir et le voyage en mer propose à cette âme sensible qui explore les nouveaux territoires de la mélancolie une infinité d'expériences qu'elle n'aurait pu faire sur la terre ferme.

\section{"Les matelots brûlés ou du moins noyés " ou Qu'être homme, c'est être capable de s'attendrir}

L'expérience du voyage en mer, c'est aussi celle du combat sur l'eau. Robert Challe en fit l'expérience dès juillet 1690 dans l'attaque, par son escadre, d'un navire anglais, le Philip Harbert de Londres, "l'un des plus beaux et des plus forts navires qui fussent à la mer $^{16}$ ». Le récit que fait Challe de cette bataille à mort est singulier en ce qu'il prend d'abord les couleurs d'une description d'opéra pour conduire à une réflexion sur l'idée de compassion. Comme souvent chez Challe, l'expérience vécue est analysée à la lumière des connaissances livresques, ici d'un souvenir d'amateur d'opéra (ce qu'il était) bien au fait de la fonction dramaturgique des décors qui est souvent, sur la scène lyrique, comme dans la tragédie grecque, de pathétiser le discours autant que d'objectiver la morale. L'expérience du combat, dans le journal de voyage, confirme ce que l'opéra faisait déjà connaître et sentir, cette idée que n'avoir pas de compassion pour la douleur, tant physique que morale, est le signe même de l' "inhumanité ", de la «barbarie ", mots d'opéra à prendre ici au sens fort. À cette occasion, c'est aussi une certaine défiance à l'égard de certains aspects de la morale héroïque qui est mise en scène ; on y reconnaît - curieusement puisqu'il s'agit de faire le récit d'un combat de type militaire - les linéaments de la morale lyrique qui ressasse sans interruption cette idée qu'être homme c'est être capable de s'attendrir.

Curieuse relation d'un combat, donc, puisque ce récit, un des moments obligés du journal qui fait en général se rejoindre récit de voyage et roman d'aventures, non seulement est saturé d'allusions à l'univers lyrique, mais encore sert d'appui à une dénonciation de l'héroïsme au profit de cette valeur douce qu'est la compassion vraie. En effet, sur le témoignage d'un fugitif français, échappé à la nage avant l'assaut, le bateau sur lequel se trouve Challe est informé de ce que le capitaine du bateau anglais « avait dit que, si nous étions Français, il se ferait plutôt brûler et sauter que de se rendre ${ }^{17}$ ». Le lecteur attend, très naturellement, une scène d'héroïsme comme on en lit dans les romans d'aventures : le capitaine ennemi, dans la seconde phase du combat surtout mené par Le Florissant, est caractérisé par son " opiniâtreté », que " tout le monde admirait ${ }^{18}$ ", " de ne se rendre pas à une force si supérieure à la sienne ${ }^{19}$ " (il se bat seul contre quatre) et il paraît, de

15 Journal..., éd. cit., « Avant-Propos », p. 12.

16 Ibid., p. 260.

17 Ibid., p. 258.

18 Ibid., p. 259.

19 Ibidem. 
surcroît, béni du ciel puisqu'il a le « bonheur de ne pas être coulé à fond, après avoir reçu tant de $\operatorname{coups}^{20}$ ». Ce sont là quelques notations liminaires typiques du récit d'aventures, mais c'est une scène d'opéra singulière qui succède à cet incipit convenu. La nuit s'étend sur la scène du combat qui s'interrompt un moment et le capitaine anglais cherche à fuir " toutes voiles dehors pour tâcher de nous échapper ${ }^{21}$ ». Et le combat se fait décor et musique. Percussion d'abord : "Nous ne fûmes plus lors que spectateurs du combat et entendions les boulets ${ }^{22}$. " Allusion claire à une mise en scène : "L'Oiseau, le plus mauvais voilier de l'escadre, parut sur la scène $»^{23}$. Ensemble instrumental ensuite, quand la ruse du capitaine échoue : "Il mit toutes voiles dehors pour tâcher de nous échapper ; mais M. de Ferrières qui voulait lui donner ce matin le premier l'aubade, comme il lui avait donné la sérénade hier, a fait aventer ${ }^{24}$. » Entrée des instruments à cordes dans la foulée : « Nous avions déjà cargué nos voiles pour faire jouer nos violons et attacher avec lui un combat réglé25. » On inclinerait à penser qu'il s'agit là d'une métaphore musicale filée sans grâce particulière, si juste après, " une bordée à peine lâchée ", ne produisait sur le bateau anglais une singulière explosion. Non celle de la salle des poudres - cela viendra ensuite -, mais celle « d'un coffre plein d'artifices, qu'on nomme ordinairement coffre à feu ", sur les navires, comme dans les théâtres à machines : on se croirait bien, en effet, dans l'univers des Torelli et des Vigarani, décorateurs autant qu'artificiers de théâtre lyrique, plutôt que dans un roman d'aventures, comme le confirme la notation en forme de didascalie qui suit : "Ce vaisseau parut tout d'un coup en feu et en flammes ${ }^{26}$. " Ensuite, le capitaine anglais, au " désespoir de pouvoir [...] défendre [son bâtiment] » met « lui-même le feu à son navire », et, en lâche patenté et non plus en héros sacrificiel, " se sauve lui-même en s'éloignant en chaloupe ${ }^{27}$."

Là commence la lecture morale de l'épisode, bien conforme à la morale de l'opéra. C'est d'abord l' " horreur ", répétée sous la forme d'une anaphore, refrain d'épouvante, qui domine, dans ce récit d'incendie sur l'eau, où l'on peut soupçonner un souvenir de l'Alcyone d'Houdar de Lamotte et Marin Marais. C'est en songeant à la scène d'évocations infernales du $\mathrm{I}^{\mathrm{er}}$ acte $^{28}$ et à celle de la célèbre tempête du quatrième, si souvent imitée, si souvent commentée, à la suite de laquelle précisément, le naufrage du bateau de Ceix plonge Alcyone dans le désespoir que lui cause cette vision d'horreur, qu'il faut

\footnotetext{
Ibidem.

1 Ibidem.

2 Ibidem.

3 Ibidem.

4 Ibid., p. 260.

5 Ibidem.

6 Ibidem.

7 Ibidem.

28 Après qu'Alcyone, Ceix et le Grand Prêtre ont ainsi commenté le déchaînement des enfers : "Quel bruit ! / Quels terribles éclats ! L'air s'allume ! Le Ciel fait gronder le tonnerre / Quel gouffre affreux s'est ouvert sous nos pas : / Tout l'enfer en courroux sort du sein de la terre ", et après que les furies sorties de enfers ont embrasé tout le décor, le chœur s’écrie : "Quel embrasement ! Quel ravage ! Dieux, injustes Dieux, quelle horreur ! ( $\mathrm{I}, \mathrm{sc} .3)$, cette scène préparant à distance, sur le plan dramaturgique et par la musique, ce grand moment de bravoure dû à Marin Marais qu'est la tempête du quatrième acte, chef-d'œuvre de musique imitative qui n'avait pas encore d'exemple sur la scène française. Cf. Recueil général des opéras représentés par l’Académie Royale de Musique (1971). Genève : Slatkine, t. II, p. 260.
} 
sans doute lire ce récit touchant qui paraît se nourrir d'une tragédie lyrique dont le sujet central est la compassion ${ }^{29}$ :

Quelle horreur de voir un navire en feu ! En un moment ce ne fut que flamme. Quelle horreur d'entendre les cris du reste de son équipage, que ce malheureux avait abandonné à une mort certaine! Quelle horreur d'entendre le mugissement des animaux, consumés tout en vie! Ce navire fut plus d'une heure et demie qu'il semblait un charbon ardent. Le feu qui sort de la fournaise n'est pas plus éclatant. Je ne crois pas qu'on puisse voir au monde pendant la nuit un spectacle plus horrible : surtout lorsqu'il eut pris aux poudres, il semblait un enfer, qui vomissait feu et flamme contre le ciel. L'air en fut tout en feu pendant un demi quart d'heure : ensuite succéda une noire et épaisse fumée, qui fut une heure à se dissiper ${ }^{30}$.

$\mathrm{Si}$ « en feu et en flammes » et « un enfer qui vomissait feu et flammes contre le ciel » font déjà songer à un enfer spectaculaire qui fleure bon la tragédie, l'antéposition des deux adjectifs qui décrivent la fumée à la fin du récit (" une noire et épaisse fumée ») signale bien une écriture instruite par une culture livresque, que nous croyons ici être nourrie des descriptions de décors en forme de didascalies qu'on lit dans les tragédies en musique.

Surtout, le spectacle infernal ouvre, dans le récit de voyage comme d'ordinaire dans l'opéra, sur un raisonnement compassionnel qui affirme d'emblée, en condamnant l'excès de bravoure et la tentation de la prouesse, que manquer de compassion, c'est, au sens propre, ne pas être humain : "C'est ainsi qu'a péri le Philip Harbert [...] et cela par l'intrépidité et l'inhumanité de son capitaine ${ }^{31}$.»

La lecture morale de l'épisode impute même à un exercice immoral de l'esprit de gloire cette absence de compassion qui rend cruel et inhumain. Challe dit le capitaine anglais «à jamais condamnable [...] par la cruauté qu'il a eue d'abandonner aux flammes et à une mort également certaine et horrible les mêmes hommes qui avaient si opiniâtrement secondé son courage et son désespoir $[\ldots]^{32}$. » Pour manifester encore un peu plus nettement où va sa compassion, Challe oppose la vie que mène le capitaine à une vignette larmoyante qu'il esquisse pour faire contraste avec la scène d'horreur qu'il vient de décrire.

29 Si Ceix incarne la compassion pour les malheureux qui périssent en mer (« Tu vois dans l'horreur des naufrages / Expirer mille malheureux », III, sc. 1, nous soulignons), c'est surtout la figure d'Alcyone qui apparaît en filigrane dans l'écriture du récit : sa crainte d'imaginer Ceix dans la douleur la conduit à le supplier de lui permettre d'embarquer à ses côtés. Elle fait ainsi à Ceix qui s'embarque cette demande : «Mon cour à chaque instant vous croira la victime / Des flots et des vents en courroux. / Je connais l'ardeur qui m'anime / Je mourrai des dangers que je craindrai toujours / [...] Consentez donc que je vous suive. » (III, sc. 4, éd. cit., p. 262.) Elle réitère plus loin cette requête encore inédite sur la scène lyrique et qui ne sera pas imitée : «Vous partez donc, cruel ! je frémis, je tremble. / Est-ce ainsi qu'à mes pleurs s'attendrit un époux ? / Laissez-moi, par pitié, m'exposer avec vous, / Du moins s'il faut souffrir, nous souffrirons ensemble. » (III, sc. 4, ibid., p. 263, nous soulignons.) L'idée d'attendrissement, centrale dans son propos, attirera l'attention de Dubos qui, dans les Réflexions critiques, fera de cet épisode l'exemple emblématique de la capacité de l'art lyrique à emporter l'adhésion de l'auditeur-spectateur qui, par l'effet immédiat de l'entre-communication du sentiment, ne saurait douter de la réalité ni de la validité, dans l'ordre du jugement esthétique, de son sentiment compassionnel. Cf. Dubos, J.-B. (1970) : Réflexions critiques sur la poésie et sur la peinture. Genève : Slatkine, IIe partie, section 45 , p. 129.

30 Journal..., p. 260.

31 Ibidem.

32 Ibidem. 
Ce bref récit en forme de tableau a été, à l'évidence, composé rétrospectivement, puisqu'il fait état d'éléments clairement postérieurs à la scène de l'incendie du navire. On y voit Robert Challe exercer sa compassion (et nous engager dans la même voie) à l'égard de la jeune épouse d'un des officiers ennemis, jeune mère dont il n'a pu entendre parler qu'après la catastrophe :

Quelque peine qu'il puisse souffrir à Amzuam, où il [le capitaine] s'est retiré, il n'est point encore tant à plaindre que la femme d'un de ses officiers qui est à terre avec deux enfants, dont il y en a un à la mamelle, en étant accouchée à bord depuis leur départ de la Tamise ; femme d'environ dix-neuf à vingt ans, qui a eu assez de résolution pour vouloir, malgré sa grossesse, suivre son mari, qui a été tué à la première bordée, et qui allait à Bombay remplir un poste de capitaine ${ }^{33}$.

Cette curieuse interruption du récit d'aventures, si l'on y songe bien, ne s'explique que si l'on comprend que Challe veut opposer à l'inhumanité du capitaine, à son héroïsme destructeur, une scène de genre qui représente, sur le mode larmoyant, comme le faisait déjà Alceste, comme Lamotte en usera dans Inès de Castro, comme le fera bientôt la peinture de chevalet, une figure féminine bien à plaindre qui oppose à la guerre, à la force brute, l'amour conjugal et l'amour maternel. Figure féminine superlative, même, qui se tient "à terre ", comme Alcyone se tient sur le rivage quand sombre avec le vaisseau dont elle contemple en songe le naufrage, son amant et donc aussi son amour et sa vie. Cette interruption émouvante dans le récit, bien faite pour attendrir le lecteur, ouvre sur un nouvel exercice conjoint de la raison et de la sensibilité dans lequel Challe s'efforce de préciser à qui doit aller la compassion la plus marquée. Sa sollicitude va d'abord aux morts, et plus particulièrement à ceux qui ont le plus souffert, et souffert plus longtemps, pour mourir : "Je ne compte plus les soldats et les matelots qui ont été tués, mais j'ai une vraie compassion de ceux qui ont été brûlés, ou du moins noyés en voulant se sauver $^{34}$. » L' " humanité tendre ${ }^{35}$ » dont Challe se dit pourvu dans les Difficultés..., qu'il décrit comme une « vertu de tempérament ${ }^{36}$ » et qu'il inscrit dans le cadre d'une morale naturelle, se révèle ici porter à d'autant plus d'attendrissement qu'elle lui fait imaginer la douleur physique d'autrui mêlée au désespoir.

Mais surtout, Challe s'attarde encore à plaindre une catégorie d'être humains à laquelle un roman d'aventures n'eût songé que comme à des chanceux ou à de francs héros : ceux qui ont pu sauver leur vie. Selon lui, en effet, ils ont nécessairement perdu dans l'aventure confiance et dans les valeurs supposées d'un héroïsme sacrificiel (où il soupçonne un dévoiement de l'amour-propre) et dans l'humanité :

Ceux qui sont à terre sont encore à plaindre. Quelle confiance peuvent-ils prendre dans un homme assez barbare pour tout sacrifier à un honneur chimérique qu'il se fait à lui-même, et ceux-mêmes auxquels il doit cet honneur qu'ils lui ont acquis par leur bravoure ${ }^{37}$.

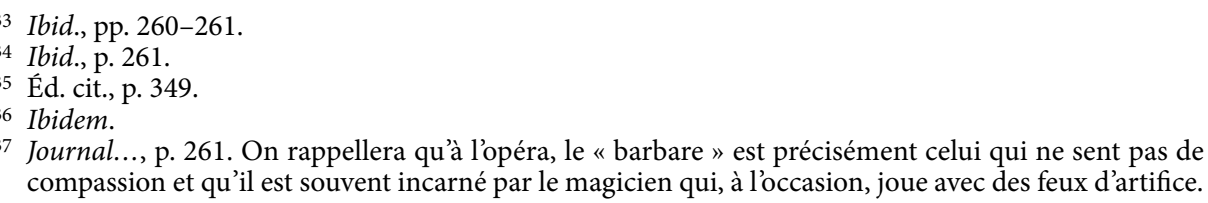
compassion et qu'il est souvent incarné par le magicien qui, à l'occasion, joue avec des feux d'artifice. 
Les plus à plaindre, ceux sur lesquels on versera, si l'on est un homme, des larmes d'attendrissement, ce ne sont pas les morts. Ce sont d'abord ceux qui ont beaucoup souffert pour mourir, ensuite, parmi ceux qui restent en vie, d'une part, ceux qui, comme la femme de l'officier, vivront privés de ceux qu'ils aimaient et qui étaient leurs appuis, de l'autre, ceux qui doivent nécessairement, dans cette cruelle expérience, avoir perdu toute confiance non seulement dans la morale héroïque, mais encore toute confiance en l'homme. La conclusion du spectacle comme du commentaire est sans appel, et l'ironie tragique sur le mot de "sauvé » le donne bien à entendre : "S'il [le capitaine] s’était brûlé lui-même, son action aurait tenu de l'héroïsme : mais il s'est sauvé, et cela lui donne une autre face ${ }^{38}$. » Le jeu sur les différents sens du mot de "sauvé » l'indique assez : si en s'enfuyant, le capitaine a bien assuré le salut de son enveloppe charnelle, loin s'en faut qu'il ait sauvé son âme. Pour tout le reste de sa vie terrestre, il s'est exclu lui-même du commerce des autres hommes et même de l'humanité tout court, à laquelle un barbare de son espèce ne saurait appartenir. Mais surtout, à l'évidence, en se sauvant, il s'est perdu, il s'est damné. Il est même devenu une figure du diable : " cela lui donne une autre face ». Challe fait d'une scène de combat typique une scène puissamment lyrique qui fait échapper le journal de voyage au pur roman d'aventures. En effet, la leçon de l'épisode comme c'est la leçon ordinaire des opéras, ce que Dubos commentant Alcyone avait bien compris, c'est bien qu'être homme, c'est être capable de s'attendrir.

Cette disposition naturelle à cette curieuse vertu-passion qu'est la compassion, et singulièrement la capacité de s'attendrir, est-elle pour autant réservée aux hommes ? Le voyage, précisément, parce qu'il confronte à une nature différente, plus riche aussi d'expériences nouvelles, ouvre à des réflexions sur l'idée de nature humaine et sur l'idée de nature sensible en général.

\section{"La guenon et son faon tués par le chasseur » ou Ce que c'est que la nature sensible.}

Si Challe a le cœur disposé de telle façon que même les petits poissons volants lui inspirent de la compassion, il fait aussi connaissance avec cette partie fascinante de la nature animale que sont les singes. Singes du Cap qu'on lui a dits « fort grands et fort amoureux des femmes et des filles ${ }^{39}$ ", singes et guenons du Cap dont on lui a dit qu'ils avaient "l'esprit de former des rendez-vous » avec des hommes et des femmes (mariées ou non), " animaux " qui " aiment avec attache ", qui sont capables d'avoir assez de sentiment pour leurs " maîtresses » humaines pour les défendre au péril de leur vie contre leurs maris jaloux et qui peuvent avoir avec des femmes une descendance qu'on "étouffe » ou que l'on «baptise » selon que le «fruit » a ou non " figure humaine et les cris d'un enfant ». De cela, Challe ne parle que par ouï-dire et, s'il incline à penser son informateur sincère, la «bestialité » (nous dirions la zoophilie) lui inspire assez de dégoût pour que sa curiosité pour ce « crime $^{40} »$ manifeste ne le porte pas à une compassion bien forte pour

38 Ibid., p. 261.

39 Ibid., p. 221.

40 Ibidem. En une seule page, à laquelle sont empruntées toutes ces petites notations au sujet des grands singes et dans laquelle il semble, par petites touches successives, rendre les expressions exactes de son 
telle femme enlevée par les singes à l'occasion d'un rendez-vous ou pour telle guenon privée de son petit, mi-singe, mi-homme, sitôt qu'elle « est accouchée ${ }^{41}$ ». Reste que ce que nous pourrions appeler, avec un soupçon d'anachronisme, la différence des espèces ne lui parait visiblement pas aussi marquée qu’à d'autres. Surtout, la séparation opérée par un Descartes entre les hommes et les animaux lui parait hautement sujette à caution : l'expérience du voyage procure à cet égard plus de sagesse vraie que la spéculation intellectuelle.

Mieux encore, l'expérience du voyage, en lui donnant l'occasion de voir, dans une circonstance touchante, une mère-singe et son petit blessés par un chasseur de la troupe, conduit Challe à proposer, en approfondissant son analyse sur la compassion, une réflexion sur l'idée de nature dont la portée tant anthropologique que métaphysique dépasse de beaucoup la controverse religieuse dont les petits poissons volants avaient été l'occasion. Si, en effet, Challe ne croit guère à l'interfécondité des singes et des humains, les corps des uns et des autres lui semblant résolument de natures distinctes, en revanche, au hasard d'une rencontre avec des sapajous, il met en scène l'idée que l'intercommunication des sensibilités touche de bien près à la communion des âmes, en quoi il s'écarte des thèses mécanistes les plus reçues.

Au cours de l'expédition, un chasseur de la troupe débarquée aux Négrades a tiré au fusil sur un sapajou, qui se révèle être une guenon qui nourrissait son petit. Elle tombe de l'arbre, blessée, son petit à côté d'elle. Le chef de l'expédition, « touché des caresses que cet animal faisait à son faon ${ }^{42}$ » décide de l'emmener pour la soigner. Elle mourra pourtant, après avoir vu dépérir son petit, le soigneur n’ayant pas réussi, malgré ses efforts, à trouver dans sa chair le dernier projectile qui l'affaiblit, lui fait endurer mille souffrances et l'empêche de nourrir son petit, chaque degré vers la consomption donnant l'occasion de petites scènes explicitement décrites comme touchantes et écrites pour toucher. Nous n'en garderons ici que le bref passage où l'idée d'attendrissement ${ }^{43}$ est absolument explicite :

Son faon mourut le troisième jour entre ses bras, faute de nourriture, le lait de sa mère étant pourri. Tout mort qu'il était, elle l'embrassa et le baisa et le mit à côté d'elle et non plus sur sa cuisse ou sur son bras, comme elle avait fait pendant qu'il avait été en vie. On la vit effectivement pleurer et on entendit dans son estomac comme des espèces de soupirs. Environ une heure après, Mr. de Porrières lui fit ôter son petit. Elle tendit les bras au matelot qui le prenait : elle le prit, le baisa de nouveau et le rendit. On lui vit encore les yeux pleins de larmes.

La Fargue vint un moment après pour la panser : elle lui baisa la main, lui montra encore avec son doigt le dessous de sa mamelle gauche; et le regarda d'un air à attendrir tous les

informateur avant d'en faire un commentaire moral, Challe donne à sa relation, tout en la constituant en vignette, la fraîcheur d'une histoire qui serait racontée de vive voix.

41 Ibidem.

42 Ibidem, t. II, p. 66.

43 Nous prenons ici la liberté de renvoyer à notre contribution au XVII Congrès de l'Association Guillaume Budé, L'Homme et ses passions, (27-28 août, 2013) où nous avons exploité ce même épisode dans une étude plus générale sur l'importance de l'idée de compassion, informée par la morale lyrique, dans la création d'un nouveau romanesque, sous le titre de "Métamorphoses de la compassion au tournant des Lumières : de l'idée lyrique d'attendrissement chez Robert Challe» (Paris : Les Belles Lettres, 2016, pp. 665-684). 
spectateurs. Je ne sais ce que Mr. de Porrières et d'autres n'auraient point donné pour sauver cette bête ${ }^{44}$.

Attendrie par la mort de son petit, la guenon est surtout attendrissante : elle tire les larmes à tout le monde et tous voudraient la voir "guérie " et même "sauvée ". Elle fournit un sujet propre à attendrir aussi le lecteur, comme en témoignent les adresses finales ${ }^{45}$. La guenon ne montre aucun signe de la sauvagerie naturelle qu'on attend d'elle. Au contraire, elle accepte les soins avec une infinie patience, elle les sollicite même, toute à son devoir de mère, si douloureux qu'ils soient, elle esquisse des gestes de supplication, manifeste sa gratitude, montre toute sa tendresse. C'est une scène à la fois théâtrale et naturelle, un tableau mélodramatique qui touche des "spectateurs » muets, interdits, et en même temps une tranche de vie donnée pour très fidèle à la réalité : Challe dit en avoir été " témoin oculaire ${ }^{46}$ ", il en précise les dates et détaille les heures. Cette scène qu'il nous propose, à nous lecteurs, nous donne à voir, à entendre, à imaginer des gestes, des soupirs, des larmes, des baisers qui disent les sentiments d'un être pourtant incapable de langage articulé. C'est un grand moment de silence et d'émotion dans le malheur partagé. On voit mis en scène et indissolublement mêlés la compassion sous les espèces de l'attendrissement, l'amour sous la forme de l'instinct de nature et l'idée, plus touchante encore, d'anéantissement. Le tableau en est riche, qui nous amène à ressentir la mort du petit singe, la compréhension de sa mort par la " mère " (qui change de manière de le porter, ne le nourrit plus, le dépose à côté d'elle, l'embrasse une dernière fois et le pleure quand on l'emporte enfin), la conscience, chez la mère, de l'imminence de sa propre mort en l'absence de « secours » efficace, puis sa mort effective, qui attriste tout le monde, y compris son soigneur, pris en flagrant délit d'ignorance et de bêtise.

Les brutes sont ici les hommes, avec leur violence à l'égard d'une pauvre créature qui nétait que tendresse au moment du coup de fusil, et avec leur peu de science, alors que la guenon montrait où il fallait sonder la plaie : l' " instinct » l'emporte sur une " raison » mal instruite, car la nature « enseigne » aux animaux, quand « Aristote, Pline, Descartes, Rohault, Gassendi, la Chambre et tous les autres qui ont donné sur les animaux leurs visions pour des vérités ${ }^{47}$ » ont parlé sans savoir et peut-être surtout sans sentir. Ce n'est pas là pour autant un simple discours sur l'âme des bêtes, sur la sensibilité des animaux, encore moins sur les droits des animaux. Outre le fait que l'expérience inflige à la théorie cartésienne de l'animal-machine un démenti incontestable, Challe présente l'émotion générale de la sensibilité comme garant d'une vérité métaphysique qui suggère un propos assez neuf sur l'individu comme sur la société des hommes : par ses larmes, la guenon leur dit, nous dit, à nous lecteurs, non pas seulement : " je suis attendrie, donc je suis ", mais, mieux encore : « je vous attendris, donc je suis ».

\footnotetext{
44 Ibidem, nous soulignons.

45 "Que le lecteur raisonne là-dessus tant qu'il lui plaira [...] Que ce lecteur me trouve parmi les femmes, j'entends les plus raisonnables, une mère qui agisse avec plus de constance, plus de tendresse et plus de fermeté pour son enfant [...]. Que le lecteur y réfléchisse à son tour. » (Ibid., t. II, p. 67.)

46 Ibidem.

47 Ibidem.
} 


\section{Conclusion}

C'est bien dans les Réflexions sur la religion proposées au Père Malebranche que Robert Challe dessine les contours d'une compassion proprement humaine, essentiellement distincte de la miséricorde divine. Mais c'est le voyage, avec ses aventures, avec ses expériences inédites, et en particulier celle du risque et de sa conséquence presque inéluctable, la douleur, qui a été l'occasion pour Challe de faire se rejoindre en une seule personne qui se découvre sensible et qui découvre ce que c'est que la nature sensible, l'homme, le philosophe (théologien à ses heures) et le littérateur. Douleur ressentie, douleur contemplée avec horreur, douleur sans cesse imaginée aussi, douleur des hommes, mais aussi douleur des bêtes, douleur intime, douleur pour soi qui s'origine aussi dans le spectacle de la douleur des autres, hommes ou singes, ou même petits poissons tombés morts d'avoir laissé sécher leurs ailes. La leçon que nous donne ce singulier aventurier qui embarqua avec, au moins dans la tête, toute une bibliothèque et quelques opéras et qui, revenu à terre, mit au net son Journal en mêlant expériences existentielles et souvenirs livresques ou musicaux, c'est au fond une leçon sur l'idée de nature dont on aperçoit la portée anthropologique et même métaphysique avec une certaine netteté si l'on confronte avec celle de Descartes et avec celle de Fontenelle son idée de ce que c'est que l'homme, son idée de ce que c'est quêtre. Grossièrement, chez Descartes le critère qui distingue ce qui est un être de ce qui n'est pas un être, c'est l'âme; chez Fontenelle, le critère qui distingue ce qui est un être de ce qui n'est pas un être, c'est ce qu'on pourrait appeler, avec les Lettres galantes, la force de "vie ", et selon ce critère il y a un monde entre un chien et une montre. Challe se montre ici clairement plus proche de Fontenelle que de Descartes, mais sans doute moins rationaliste que le chef des Modernes. A la faveur des expériences de vie permises par le voyage et par lui seul, dans un dualisme encore bien lisible qui distingue le corps, d'une part, et, de l'autre l'esprit ou le cœur dans les Difficultés, Challe fait du sentiment et plus particulièrement de cette passion ou de cette vertu qu'est la capacité non pas seulement de s'attendrir, mais d'attendrir autrui, non pas essentiellement le point de conjonction entre le corps et l'esprit, mais plus précisément, le critère de l'être. Etre au monde, être aux autres hommes, être à soi, être soi, c'est tout un pour Challe qui découvrit, peut-être mieux en mer qu'à terre, dans le quotidien et les épreuves du voyage plutôt que dans la vie ordinaire, que l'appréhension de la douleur révèle l’être humain et sa nature morale. 


\section{BIBLIOGRAPHIE}

Artigas-Menant, G. (dir.) (2008) : Robert Challe et les passions. Préfacé par F. Deloffre. Paris : PUPS

Challe, R. (1959) : Les Illustres Françaises. Édition établie par F. Deloffre. Paris : Les Belles Lettres, 2 vol., $3^{\mathrm{e}}$ tirage, 1973 .

Challe, R. (1983) : Journal d'un Voyage fait aux Indes Orientales. Édition établie par F. Deloffre et M. Menemencioglu. Paris : Mercure de France, 2 vol.

Challe, R. (2000) : Difficultés sur la religion proposées au Père Malebranche. Édition nouvelle d'après le manuscrit complet et fidèle de la Staatsbibliothek de Munich par F. Deloffre et F. Moureau. Genève : Droz.

Dubos, J.-B. (1970) : Réflexions critiques sur la poésie et sur la peinture. Genève : Slatkine.

Guyon-Lecoq, C. (2016) : « Métamorphoses de la compassion au tournant des Lumières : de l'idée lyrique d'attendrissement chez Robert Challe ", in L'homme et ses passions (2016) : Actes du XVII congrès de l’Association Guillaume Budé. Textes réunis par Boehm, I. - Ferrary, J.-L. - Franchet d’Espéray, S. Paris : Guillaume Budé.

Recueil général des opéras représentés par l’Académie Royale de Musique. (1971). Genève : Slatkine.

Camille Guyon-Lecoq

CERCLL (Centre d'Etudes des Relations et Contacts Linguistiques et Littéraires)

Université de Picardie Jules Verne

camille.guyon-lecoq@u-picardie.fr 